\title{
ORIGINAL ARTICLE Phosphorylation of SOS1 on tyrosine 1196 promotes its RAC GEF activity and contributes to BCR-ABL leukemogenesis
}

$\mathrm{S} \mathrm{Gerboth}^{1,9}$, E Frittoli $^{1,9}$, A Palamidessi ${ }^{1}$, FC Baltanas ${ }^{2,10}$, M Salek ${ }^{1,11}$, J Rappsilber ${ }^{1,12}$, C Giuliani ${ }^{1}$, F Troglio ${ }^{1}$, Y Rolland $^{1}$, G Pruneri $^{3}$, S Kreutmair ${ }^{4}$, I Pallavicini ${ }^{3}$, M Zobel ${ }^{1}$, M Cinquanta ${ }^{5}$, S Minucci ${ }^{3,4}$, C Gomez $^{2}$, E Santos ${ }^{2}$, AL Illert ${ }^{6,7}$ and G Scita ${ }^{1,8}$

Son of Sevenless 1 (SOS1) is a dual guanine nucleotide exchange factor (GEF) that activates the small GTPases RAC and RAS. Although the molecular mechanisms of RAS GEF catalysis have been unveiled, how SOS1 acquires RAC GEF activity and what is the physio-pathological relevance of this activity is much less understood. Here we show that SOS1 is tyrosine phosphorylated on Y1196 by ABL. Phosphorylation of Y1196 controls SOS1 inter-molecular interaction, is required to promote the exchange of nucleotides on RAC in vitro and for platelet-derived growth factor (PDGF) activation of RAC- and RAC-dependent actin remodeling and cell migration. SOS1 is also phosphorylated on Y1196 by BCR-ABL in chronic myelogenous leukemic cells. Importantly, in these cells, SOS1 is required for BCR-ABL-mediated activation of RAC, cell proliferation and transformation in vitro and in a xenograft mouse model. Finally, genetic removal of Sos 1 in the bone marrow-derived cells (BMDCs) from Sos $1^{\text {fl/fl }}$ mice and infected with BCR$A B L$ causes a significant delay in the onset of leukemogenesis once BMDCs are injected into recipient, lethally irradiated mice. Thus, SOS1 is required for full transformation and critically contribute to the leukemogenic potential of BCR-ABL.

Leukemia (2018) 32, 820-827; doi:10.1038/leu.2017.267

\section{INTRODUCTION}

Son of Sevenless 1 (SOS1) has a central role in signal transduction from receptor tyrosine kinases (RTKs) to small $G$ proteins RAS and RAC. ${ }^{1-4}$ Structural and biochemical studies revealed that SOS1 is a multi-domains protein with extensive intramolecular interaction that tightly constrains its activity. ${ }^{5}$ For example, SOS1 possesses two binding sites for RAS, one of which is an allosteric site that is distal to the active one. ${ }^{6}$ Binding of active RAS at this site stimulates the nucleotide-exchange activity of SOS1 by causing conformational changes that allow substrate RAS to bind. ${ }^{2,7-9}$

An additional important domain is the $\mathrm{DH}-\mathrm{PH}$ (Dbl and Pleckstrin Homology domain), which is a structural hallmark for exchange factors of the Rho family of GTPases, and was proposed to act as a RAC-specific guanine nucleotide exchange factor (GEF). ${ }^{10-20}$ However, the DH-PH unit is folded into a closed conformation, implying that SOS1 RAC GEF activity is autoinhibited and requires specific molecular events to be activated. ${ }^{8,21,22}$ Two distinct mechanisms of SOS1 activation have been proposed: SOS1 must assemble into a multimolecular complex with EPS8, ABI1 to become catalytically active. ${ }^{12,17-20}$ The second mechanism, instead, involves tyrosine phosphorylation of SOS1 by $\mathrm{ABL}$ kinase. $^{15}$ However, how tyrosine phosphorylation induces SOS1 RAC GEF activity and its pathophysiological relevance remains ill defined.

Physiologically, ABL kinase participates in RTK-induced actin cytoskeleton remodeling, a signaling pathway in which the function of the SOS1-RAC axis is pivotal. ${ }^{15}$ Hyperactive, uncontrolled kinase activity of $A B L$ is also responsible for its oncogenic potential. The p210-BCR-ABL fusion oncoprotein is indeed necessary and sufficient to cause chronic myelogenous leukemia. ${ }^{23,24}$ Although the role of BCR-ABL in chronic myelogenous leukemia has been well studied, the critical signaling pathways mediating its transforming ability are incompletely clarified. Among them, the one leading to the activation of RAC GTPases critically mediates BCR-ABL transformation. ${ }^{25-27}$ Indeed, RAC3, one of the three mammalian RAC isoforms, is activated in p190-BCR-ABL malignant precursor B-lineage lymphoblast and its removal increases survival in p190-BCR-ABL-transgenic, RAC3-null mice (a model of acute lymphoid leukemia). ${ }^{28}$ In addition, the ubiquitously expressed RAC1 and hematopoietic-restricted RAC2 were shown to be critical for p210-BCR-ABL-mediated leukemogenesis and myeloproliferative disease. ${ }^{29}$ How RAC becomes activated by $A B L$ thus becomes a key question to be addressed. SOS1, which is expressed in myeloid cells and is regulated by $A B L,{ }^{15}$ may serve this role.

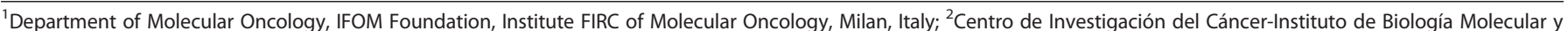

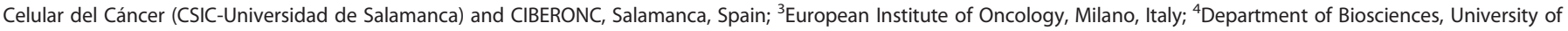

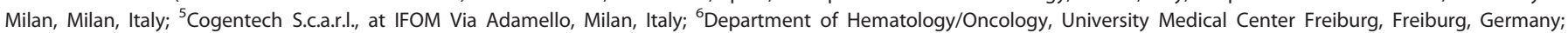

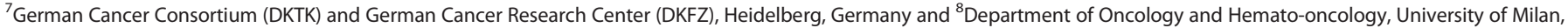

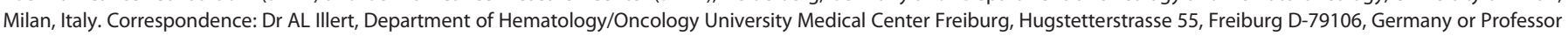
G Scita, Department of Molecular Oncology, IFOM Foundation, Institute FIRC of Molecular Oncology, Via Adamello, 16, Milan 20139, Italy.

E-mail: lena.illert@uniklinik-freiburg.de or giorgio.scita@ifom.eu

${ }^{9}$ These authors contributed equally to this work.

${ }^{10}$ Current address: Department of Neurosciences, University of California San Diego, 9500 Gilman Drive, La Jolla, CA 92093-0662, USA.

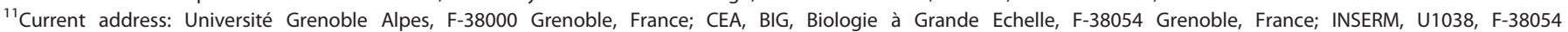
Grenoble, France.

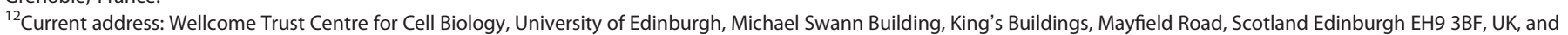
Institute of Biotechnology, Technische Universität Berlin, Gustav-Meyer-Allee 25, Berlin 13355, Germany.

Received 22 June 2017; revised 7 August 2017; accepted 11 August 2017; accepted article preview online 18 August 2017 ; advance online publication, 12 September 2017
} 
Here we show that phosphorylation of Y1196 on the C-terminal proline-rich region of SOS1 is sufficient to elicit its RAC GEF activity in vitro and in vivo in response to the activation of various receptor and non-RTKs, including ABL. We further demonstrate that Y1196 of SOS1 is phosphorylated in BCR-ABL leukemic human and murine blasts, and is required for full RAC activation, cell proliferation and transformation in vitro and in mouse models. Genetic removal of SOS1 delays the onset of BCR-ABL leukemogenesis.

\section{MATERIALS AND METHODS}

Materials and methods are described in details in Supplementary Information.

\section{RESULTS}

SOS1 is tyrosine phosphorylated on Y1196 in vitro

To investigate the molecular, biochemical and functional consequences of $A B L$-mediated tyrosine phosphorylation of SOS1 we sought to identify the target tyrosine(s). As an initial approach, we subjected full-length SOS1 and various recombinant shorter fragments to an in vitro kinase assay with $A B L$ kinase (Supplementary Figure S1A (top)). Only the fragment encompassing the C-terminal proline-rich region was efficiently tyrosine phosphorylated (Supplementary Figure S1A), hence restricting the region targeted by $A B L$.

Next, we used in vitro tyrosine phosphorylated SOS1 and titanium oxide-based affinity chromatography to enrich for phosphorylated peptides coupled with nano liquid chromatography-tandem mass spectrometry analysis ${ }^{30,31}$ (Figure 1a). We generated a peak list, which was searched against the IPI human database using Mascot 2.0 (Matrix Science Inc., Boston, MA, USA). However, no pY-containing peptide could be identified automatically by Mascot search, but spotted instead by searching the MS/MS data set for pTyr immonium ion at $\mathrm{m} / \mathrm{z} 216.043^{\text {(refs } 32,33 \text { ) }}$ and subsequently sequenced manually. This procedure revealed Y1196, located in the proline-rich C-terminus of SOS1, as the major putative $A B L$ kinase target (Figure 1a). We validated this result using two-dimensional phospho-peptide mapping that revealed a
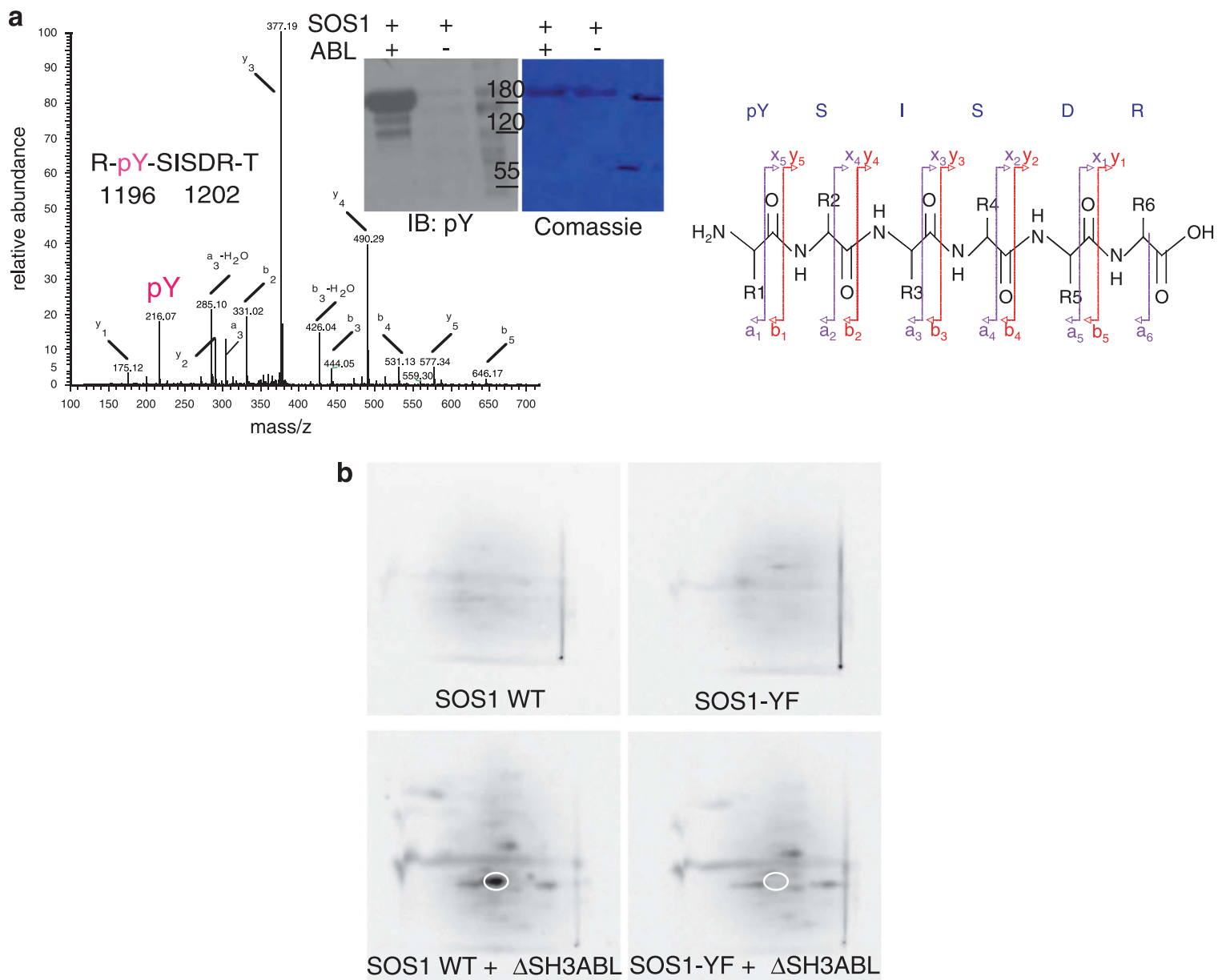

Figure 1. Tyrosine 1196 of SOS1 is the major target residue of ABL kinase. (a) SOS1 immuno-purified from SOS1-expressing 293T cells (1 mg lysates) with an anti-SOS1 antibody was in vitro phosphorylated with ABL kinase, resolved on SDS-Page and detected by Coomassie staining or by immunoblotting (IB) using the indicated antibody. SOS1 band was digested with trypsin. Phosphorylated peptides were enriched with titanium oxide beads and analyzed by tandem mass spectrometry. Left: the diagram shows the detected fragments of the phosphorylated pYSISDR peptide. Fragments are presented according to their mass/charge ratio $(\mathrm{m} / \mathrm{z})$ and their intensity (relative abundance). The intensity of the highest peak corresponds to a relative abundance of $100 \%$. Right: The possible fragmentation of the peptide is shown. The peak ( $\mathrm{pY}$ ) in the diagram (left) with an $\mathrm{m} / \mathrm{z}$ of 216,07 corresponds to the $a_{1}$ fragment on the right. Mw markers are indicated on the side of the immunoblots. (b) WT-SOS1 and Y1196F-SOS1 immunopurified from SOS1-overexpressing 293T cells (1 mg lysate) were incubated in the presence or absence (Control) of ABL kinase and ${ }^{32}$ P-ATP and subjected to SDS-polyacrylamide gel electrophoresis. After PonceauS staining, the SOS1 band was excised and digested with trypsin. Oxidized peptides were separated by electrophoresis and thin layer chromatography. 
single, major phosphorylated spot derived from WT-SOS1, but not from Y1196F-SOS1 mutant (Figure 1b). Thus, tyrosine 1196 is the major ABL phosphorylation site of SOS1 in vitro.

\section{SOS1 is tyrosine phosphorylated on Y1196 in vivo}

To assess whether Y1196 is phosphorylated in vivo, we undertook two distinct approaches. First, we ectopically expressed WT-SOS1 or Y1196F-SOS1 mutant in combination with an activated form of $\mathrm{ABL}(\mathrm{ABL}-\triangle \mathrm{SH} 3)^{34}$ in $293 \mathrm{~T}$ cells. Immunoblotting with anti-pY antibodies of SOS1 immunoprecipitates revealed that tyrosine phosphorylation of Y1196F-SOS1 was significantly reduced with respect to WT-SOS1 (Supplementary Figure S1C). Next, we raised monoclonal, anti-pY1196 antibodies the specificity of which was tested using WT- and Y1196-SOS1 in various assays (Supplementary Figures S2A and B). We detected Y1196 phosphorylation in lysates of cells expressing activated $A B L$ and WT-SOS1, but not Y1196F-SOS1 mutant, both when we immunoprecipitated total SOS1 with anti-hemagglutinin (HA) antibody, followed by immunoblotting with anti-pY1196 or vice versa (Supplementary Figure S3A). In addition, as SOS1 and ABL act on RTK-dependent signaling pathways, ${ }^{15,35,36}$ we found that plateletderived growth factor (PDGF) stimulation efficiently phosphorylated endogenous SOS1 on Y1196 in mouse embryo fibroblasts (MEFs) (Supplementary Figure S3B). Similarly, stimulation of ERBB family members with either Heregulin or epidermal growth factor in breast cancer cell lines increased Y1196-SOS1 phosphorylation, which was abrogated by the ABL kinase inhibitor Imatinib (STI571) (Supplementary Figure S3B). We concluded that phosphorylation of Y1196 occurs physiologically in response to a variety of RTKdependent stimuli, presumably through $A B L$ kinase activity.

Phosphorylation of Y1196 is sufficient to promote the exchange of nucleotides on RAC1, but not on H-RAS in vitro

Next, we tested GEF activities on RAC1 and H-RAS of tyrosinephosphorylated SOS1. Tyrosine-phosphorylated SOS1, purified from cells co-expressing activated ABL, but not Y1196F-SOS1 mutant, displayed detectable RAC1-GEF activity in in vitro assays (Supplementary Figures S4A and B). Importantly, under the same conditions, WT- and Y1196F-SOS1 showed similar levels of H-RASGEF activity, indicating that tyrosine phosphorylation specifically affects RAC1, but not H-RAS-GEF activity (Supplementary Figure S4A). To confirm that the $\mathrm{Y} 1196 \mathrm{~F}$ mutation abrogates ABL-mediated SOS1 RAC1 GEF activity, we performed a double affinity purification of SOS1 from lysates of cells expressing activated ABL and SOS1 using a GRB2-GST column in tandem with an anti-pY1196 antibody-protein G-sepharose column (Supplementary Figure S4C). The final elution step with phosphorylated antigenic SOS1 peptides yielded highly purified pY1196-SOS1 (Supplementary Figure S4C). Using a fluorescentnucleotide-based RAC1-GEF assay, we showed that pY1196-SOS1 promoted guanine nucleotide exchange on RAC1 at catalytic concentrations, similar to the DH-PH domain of TIAM1, used as positive control (Figure 2a). Finally, dephosphorylation of pY-SOS1 with a tyrosine-specific phosphatase abrogated its RAC1-GEF activity (Figure 2b). Thus, tyrosine-phosphorylated SOS1 acts as a RAC1-GEF in vitro.

SOS1 inter-molecular interaction is inhibited by activated $A B L$

These findings raise the question as to the underlying molecular mechanism. Crystallization studies revealed that SOS1 is engaged into extensive intra-molecular and possibly inter-molecular interactions. ${ }^{8,37}$ However, none of the solved structures of SOS1 includes the proline-rich region, which contains Y1196. We hypothesized that also this region undergoes intramolecular or intermolecular interactions with other SOS1 domains. We initially tested the ability of various, immobilized SOS1 fragments to bind the soluble proline-rich region in far western assays. We found that SOS1 proline-rich region binds with a micromolar (apparent) affinity to its $\mathrm{DH}-\mathrm{PH}$ domain and further restricted the region of interaction to the PH domain (Supplementary Figures S5A-C). The SOS1 $\mathrm{PH}$ domain has been proposed to exert an inhibitory effect on the $\mathrm{DH}$ domain by blocking its accessibility to RAC1. ${ }^{22}$ A significant change in the fold must therefore be invoked for this domain to become catalytically active. Loss of binding of the proline-rich region to the $\mathrm{DH}-\mathrm{PH}$ domain may be implicated in initiating these structural changes. We thus performed a binding assay between the DH-PH domain and either the phosphorylated or a non-phosphorylated Y1196-containing peptide. The phosphorylated peptide bound the $\mathrm{DH}-\mathrm{PH}$ domain with a much lower apparent affinity $\left(K_{d}>200 \mu \mathrm{M}\right)$, as compared with the nonphosphorylated peptide ( $K_{\mathrm{d}} \sim 30 \mu \mathrm{m}$ ) (Supplementary Figure S5D). These findings are also compatible with the possibility of trans rather than cis interaction between two SOS1 molecules, where the proline-rich region of one molecule may bind to the $\mathrm{DH}-\mathrm{PH}$ domain of the other. If this was the case, then a fraction of SOS1 may form dimers, as previously suggested, ${ }^{37}$ whose stability should be controlled by Y1196 phosphorylation. Consistently, by exploiting differentially HA- and green fluorescent protein (GFP)- a

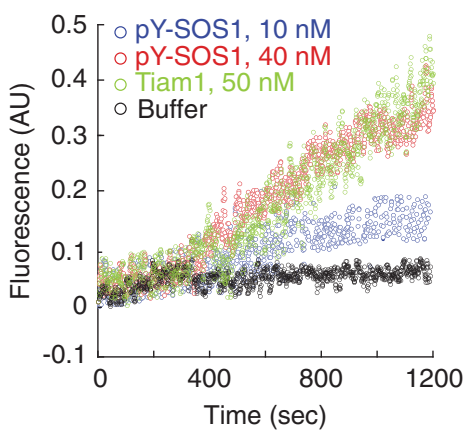

b

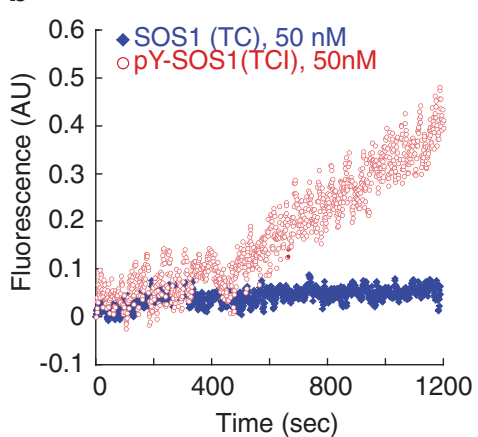

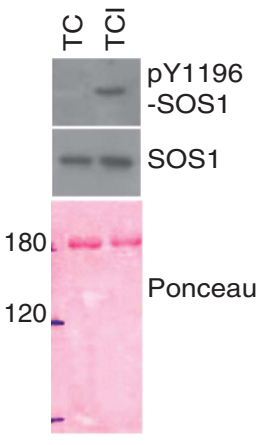

Figure 2. Phosphorylation of Y1196 by ABL is sufficient to elicit SOS1 RAC GEF activity. (a and b). MANT-GDP-fluorescence RAC GEF assay of purified pY1196F-SOS1. GST-RAC1 was equilibrated with $2 \mu \mathrm{m}$ MANT-GDP. After $300 \mathrm{~s}$, we added: (b) purified, phospho-enriched SOS1 (30 $\mu$ l and $120 \mu \mathrm{l}$, corresponding to 10 and $40 \mathrm{~nm}$, respectively) or $50 \mathrm{~nm}$ of His-DH-PH of Tiam 1 (used as positive control). Buffer indicates the nonstimulated nucleotide exchange rate of RAC1; left panels, purified, phospho-enriched SOS1 or un-phosphorylated SOS1 (treated with T-cellspecific tyrosine phosphatase either in the presence (TCI) or the absence (TC) of T-cell phosphatase inhibitor). The reaction containing MANTGDP was excited at $360 \mathrm{~nm}$. The emission of MANT-GDP-bound to RAC was measured at $440 \mathrm{~nm}$. Right panels: aliquots of phosphorylated (TCI) and phosphatases dephosphorylated (TC) SOS1 was immunoblotted with the antibodies indicated on the right. 
tagged SOS1 constructs, we showed that: (i) a sizable fraction of HA-tagged SOS1 co-immunoprecipated with GFP-SOS1 (Supplementary Figure S5E); (ii) the concomitant expression of an activated $A B L$ robustly reduced this inter-molecular interaction (Supplementary Figure S5E); and (iii) mutation of Y1196F that prevents $\mathrm{ABL}$-mediated phosphorylation and the acquisition of RAC1-GEF activity also inhibited the inter-molecular interaction (Supplementary Figure S5F). These results indicate that SOS1 needs to be in its tyrosine phosphorylated, monomeric form to act as RAC1 GEF. A corollary of this mode of action is that a SOS1Y1196F mutant should not only be deficient in RAC1 activation in vitro (as we showed above), but may also act as a dominant negative. Consistently, the expression of SOS1-Y1196F completely inhibited RAC1-GTP levels in SOS1 proficient cells, acting in a dominant-negative manner (Supplementary Figure S5G).

Although more work is needed to precisely decipher the sequence of molecular events, the sum of this finding indicates that tyrosine phosphorylation of $\mathrm{Y} 1196$ promotes the dissociation of SOS1 inter-molecular interaction, resulting in the formation of monomers which, either alone or through subsequent incorporation into larger molecular weight complexes (see Discussion), leads to optimal activation of SOS1 RAC1-GEF.

Phosphorylation of SOS1 Y1196 is required for optimal PDGFdependent activation of RAC1, RAC1-dependent actin remodeling and migration

To validate the physiological relevance of Y1196 phosphorylation of SOS1 in controlling the activation of RAC, we measured the cellular levels of GTP-bound RAC. Initially, we used cells coexpressing either WT- or Y1196F-SOS1 together with activated $A B L$. Activated ABL increased RAC1-GTP levels, which are not significantly altered by the concomitant expression of SOS1, indicating that the latter protein is not a limiting factor in this pathway (Supplementary Figure S6A). Conversely, co-expression of activated $A B L$ and $Y 1196 \mathrm{~F}-S O S 1$ robustly reduced $A B L-$ mediated RAC activation (Supplementary Figure S6A), consistent with Y1196F-SOS1 exerting a dominant-negative function.

Next, we assessed whether this posttranslational modification had an impact on RAC activation in response to PDGF stimulation ${ }^{15,35}$ using Sos $1-/-$ MEFs reconstituted with either WT- or Y1196F-SOS1 (Supplementary Figure S6B). Sos1 - / - MEF showed reduced RAC-GTP levels as compared with Sos 1- / - MEF reconstituted with WT-SOS1 (Figure 3a). Conversely, the expression of Y1196F-SOS1 did not restore RAC activation (Figure 3a). Notably, PDGF-induced ERK and AKT activities were unaffected by either SOS1 removal or the expression of Y1196F-SOS1 mutant (Supplementary Figure S6C). In agreement with the observation that pY1196 of SOS1 mediates not only RAC activation, but also PDGF-induced, RAC-dependent actin remodeling, Sos 1-/- MEFs were impaired in the formation of PDGF-induced circular dorsal ruffles (Figure 3b), where SOS1 localizes (Supplementary Figure S6D). Circular dorsal ruffles are RAC-dependent migratory and endocytic structures that mark the acquisition of a mesenchymal mode of cell locomotion. ${ }^{38}$ This response was restored by WT-SOS1, but not Y1196F-SOS1 (Figure 3b). Notably, Y1196F-SOS1 expression not only failed in rescuing circular dorsal ruffle, but also inhibited their formation (Figure $3 \mathrm{~b}$ ). The specific requirement of SOS1 as a RAC-GEF in PDGF-induced actin remodeling is underscored by the finding that functional interference with VAV, a RAC-GEF family protein regulated by tyrosine phosphorylation, had no effect on circular dorsal ruffle formation (Supplementary Figure S6E). Finally, Sos 1-/- MEFs were impaired in cell motility, which was increased by the expression of WT, but not of Y1196-SOS1 (Supplementary Figure S6F and Supplementary Movie S1). Thus, SOS1 and phosphorylation of Y1196 are required for optimal RAC1 activation
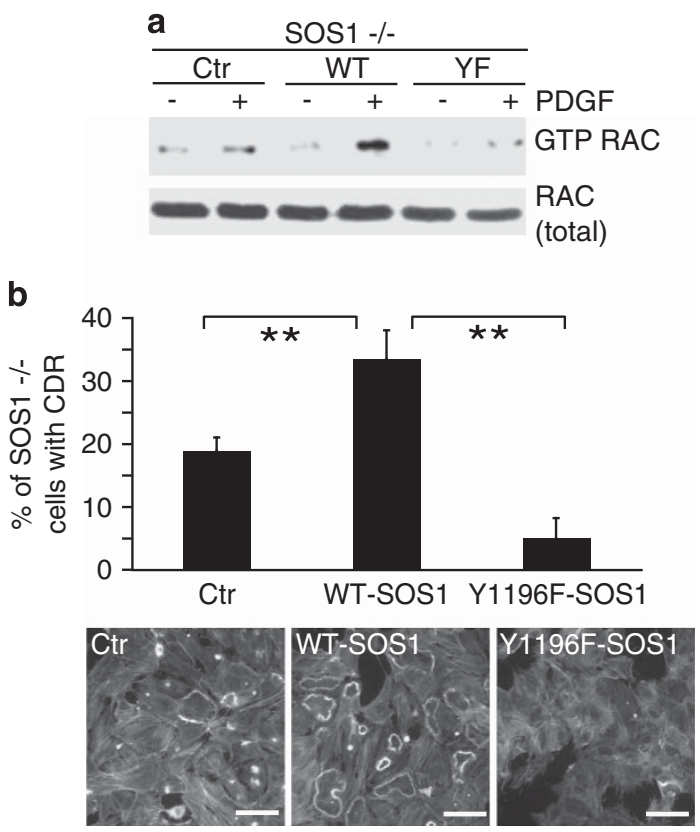

Figure 3. Phosphorylation of SOS1-Y1196 is required to promote optimal RAC activation, RAC-dependent actin remodeling in fibroblasts in response to PDGF. (a) Wild type, but not Y1196FSOS1, reconstitutes RAC activation in Sos 1 - / - MEFs. Serum-starved Sos 1 - / - MEFs, reconstituted with HA-WT-SOS1 or HA-Y1196F-SOS1 (YF) or empty vector, as control (Ctr) (see Supplementary Figure S6A), were stimulated with $10 \mathrm{ng} / \mathrm{ml}$ of PDGF for $7 \mathrm{~min}$. Activated RAC-GTP was pulled down from $1 \mathrm{mg}$ of total lysates using $20 \mu \mathrm{g}$ of recombinant GST-CRIB. Lysates $(20 \mu \mathrm{g})$ and bound proteins were immunoblotted with the indicated antibodies. (b) Serum-starved Sos 1-/- MEFs, reconstituted with HA-WT-SOS1 or HA-Y1196F-SOS1 or empty vector (Ctr), were stimulated with $10 \mathrm{ng} / \mathrm{ml}$ of PDGF for $7 \mathrm{~min}$. Cells were stained for F-actin with TritcPhalloidine. The number of cells with circular dorsal ruffle (CDR) is expressed as percent of total. At least 100 cells were scored for each condition. The data represents the mean \pm s.e.m $(n=4$ performed in triplicates). Right panels: representative pictures of MEFs forming CDR. Scale bar, $40 \mu \mathrm{m}$.

and RAC1-dependent actin remodeling and cell motility in response to PDGF stimulation.

SOS1 is phosphorylated by oncogenic BCR-ABL in leukemic blast and is required for BCR-ABL-mediated full transformation

We next assessed whether phosphorylation of SOS1 Y1196 might also be critical in pathological conditions characterized by a hyperactive $A B L$ kinases activity. The fusion oncoprotein BCR-ABL originating from a balance chromosome translocation leading to chronic myelogenous leukemia is a case in point. ${ }^{39}$ Among BCR$A B L-s t i m u l a t e d$ oncogenic pathways the one leading to activation of RAC proteins has been shown to be pivotal for full transformation. ${ }^{25,29,40}$ We thus tested whether SOS1 contributes to BCR-ABL-mediated RAC activation and transforming potential. Using pY1196-phospho-specific antibody, we showed that SOS1 is tyrosine phosphorylated in K562, a human cell line derived from a chronic myelogenous leukemia patient, and in BCR-ABLexpressing murine hematopoietic progenitor 32D cell line (Supplementary Figures S7A and B). The administration of the ABL kinase inhibitor STI571 abolished SOS1 phosphorylation in both cell lines (Supplementary Figure S7B). Next, we generated K562 cells stably knocked down for SOS1 (SOS1-KD) with three independent short hairpin RNAs (see also Supplementary Methods). SOS1 silencing reduced RAC-GTP levels, similar to 

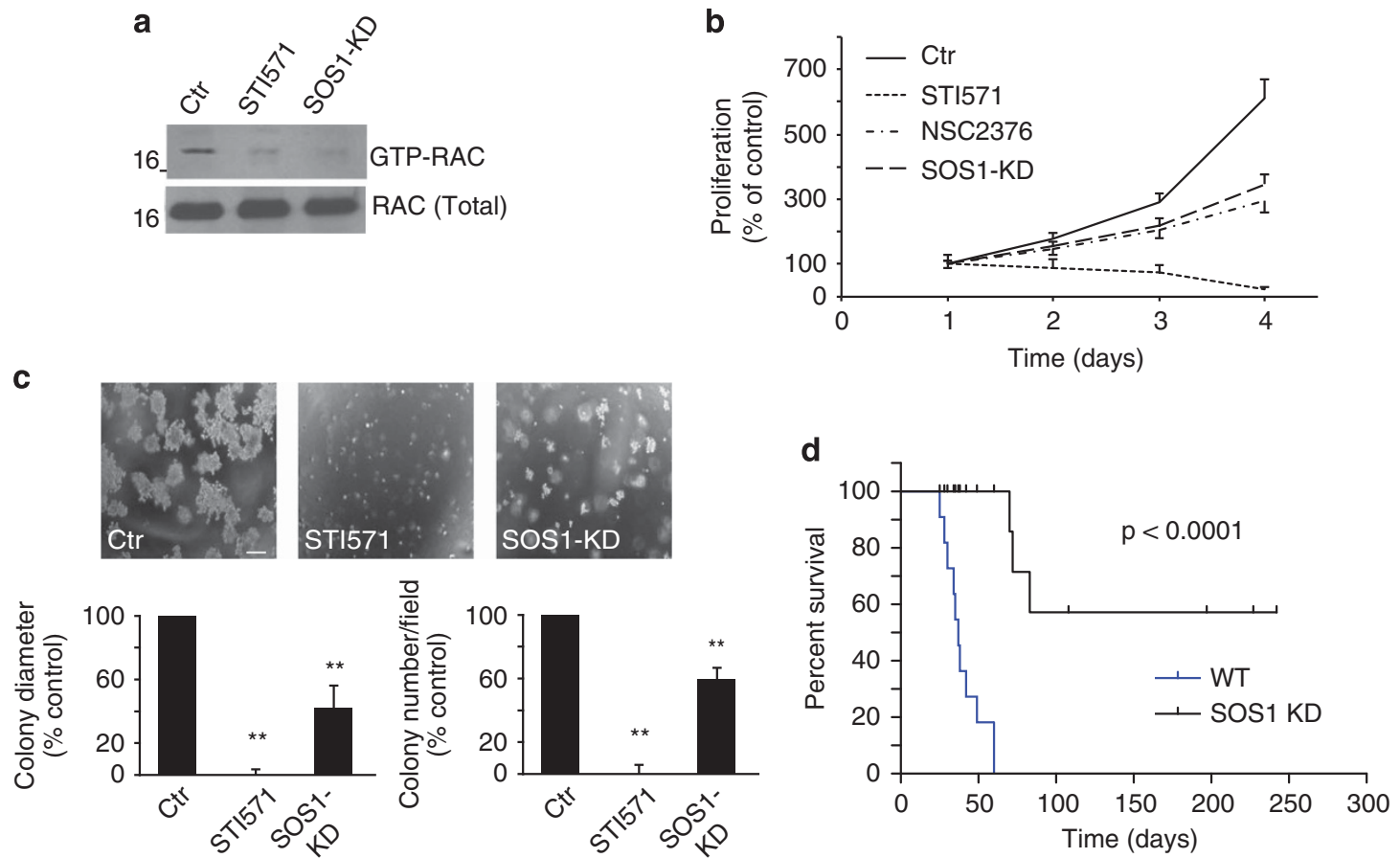

Figure 4. SOS1 phosphorylation is required for RAC1 activation and BCR-ABL-mediated full transformation. (a) K562 cells infected with pSuperRetroPuro Control vector (Ctr) or pSuperRetroPuro SOS1 interfering vector (SOS1-KD) were treated for 10 min with $10 \mu \mathrm{M}$ STI571 or vehicle as control (Ctr). We used three independent short hairpin RNA (shRNA) to generate three mass SOS1-KD populations (see Supplementary Methods), which gave similar results. Cell Lysates were incubated for $1 \mathrm{~h}$ with $20 \mu \mathrm{g}$ of GST-CRIB to pull down GTP-loaded RAC and immunoblotted to detect activated RAC. (b) An equal number (50 000 cells/ml) of K562 control cells (Ctr), STI571-treated, NSC2376treated or SOS1-KD cells (SOS1 KD) were plated and counted at the indicated times. Data are the mean \pm s.e.m. of four independent experiments done in triplicates. (c). An equal number (100 000 cells/ml) of control (Ctr), or STI571-treated or SOS1-KD K562cells (SOS1 KD) were plated in soft agar. Upper panels, representative pictures of the colonies obtained after 14 days. Lower graphs, quantification of colony number (left) and colony diameter (right). At least 50 colonies were counted. Data are the mean \pm s.e.m ( $n=3$ independent experiments). ${ }^{* *} P<0.005$ Student's $t$-test. Scale bar, $200 \mu \mathrm{m}$. (d) An equal number $\left(5 \times 10^{5}\right)$ of control and SOS-1 KD K562 were injected into the tail vein of immunodeficient mice. Mice were monitored for survival ( $n=15$ of a representative experiment run in duplicate). $P$-values (Pearson) were measured by $x^{2}$-test.

STI571 treatment (Figure 4a), but had no impact on ERK1/2 activity (Supplementary Figure S7C). In addition, SOS1-KD K562 cells had a significantly reduced rate of proliferation compared with scramble-transfected control cells (Figure $4 \mathrm{~b}$ ), but similar to the one achieved by treatment with the RAC inhibitor, NSC27633 (Figure 4b). Notably, treatment with STI571 was, as expected, ${ }^{39}$ more effective in reducing cell proliferation (Figure $4 \mathrm{~b}$ ), also as a consequence of increase cell death, which instead was not observed after SOS1 removal or RAC inhibition (Supplementary Figure S7D). The slower proliferation rate of SOS1-KD K562 cells was also accompanied by increased expression of hemoglobin, a prototypical erythroid marker (Supplementary Figure S7E).

Next, we tested weather SOS1 ablation impairs transformation and tumorigenesis. In soft agar growth assays, under conditions in which STI571 treatment completely abrogated transformation, SOS1 silencing significantly reduced both the number and the size of colony as compared with untreated, control K562 cells (Figure 4c). In xenotransplantation mouse model, NOD/SCID mice injected with control K562 rapidly died due, as expected (Figure 4d), to the expansion of leukemic blasts (not shown). The removal of SOS1 from K562 cells drastically increased mice survival (Figure $4 d$ ).

To provide direct evidence that the above phenotypic alterations are caused by the loss of Sos1 and of Y1196 phosphorylation, we restored the expression of either WT- or Y1196F-SOS1 mutant into SOS1-KD K562 cells using short hairpin RNA-resistant variants fused to GFP. GFP-WT and GFP-Y1196FSOS1 expression levels were comparable to those of endogenous
SOS1 of control K562, whereas endogenous SOS1 levels in the ectopically reconstituted cells remain significantly downregulated (Supplementary Figure S8A). Re-expression of WT-SOS1, but not of Y1196F-SOS1 mutant, reduced hemoglobin, suggesting that differentiation blockade was restored (Supplementary Figure S8A), restored RAC-GTP levels (Supplementary Figure S8B), cell proliferation (Supplementary Figure S8C) and transformation potential (Supplementary Figure S8D).

The transient nature of SOS1 knocked down and Y1196F reexpression, which were counter selected and lasted only for a limited number of passages (similar data were obtained using CRISPR-SOS1-KO K562 cells-not shown), prevented us to assess whether also in vivo we could restore leukemogenesis. To overcome this limitation, we employed a murine model in which the loss of Sos 1 can be induced by tamoxifen-mediated CRE recombination (herein on referred to as $\operatorname{Sos} 7^{\mathrm{fl}} \mathrm{fl}^{\mathrm{f}}$ ). ${ }^{41}$ bone marrowderived cell (BMDC) from control and $\operatorname{Sos} 7^{\mathrm{fl}} / \mathrm{fl}^{\mathrm{fl}}$ mice were retrovirally infected with EGFP-p210-BCR-ABL-fusion gene-expressing retroviral vectors (MigBCR-ABLp ${ }^{210}$ ), treated with tamoxifen to induce Sos 1 deletion and tested in vitro and in vivo to assess BCR$A B L$ transforming and leukemogenic ability (Figure 5). Consistent with the results obtained with human $\mathrm{K} 562$ cells, genetic removal of Sos 1 significantly reduced colony formation (Supplementary Figure S9A), without altering the immunophenotypes of BCR-ABLtransformed Sos1 null BMDC as compared with control cells (Supplementary Figure S9A). More relevantly, tamoxifen-mediated Sos 1 loss of BCR-ABL-infected Sos $1^{\mathrm{fl}} / \mathrm{fl}^{\mathrm{ll}}$ BMDC significantly delayed the development of leukemia upon BMDC transplantation into 
a
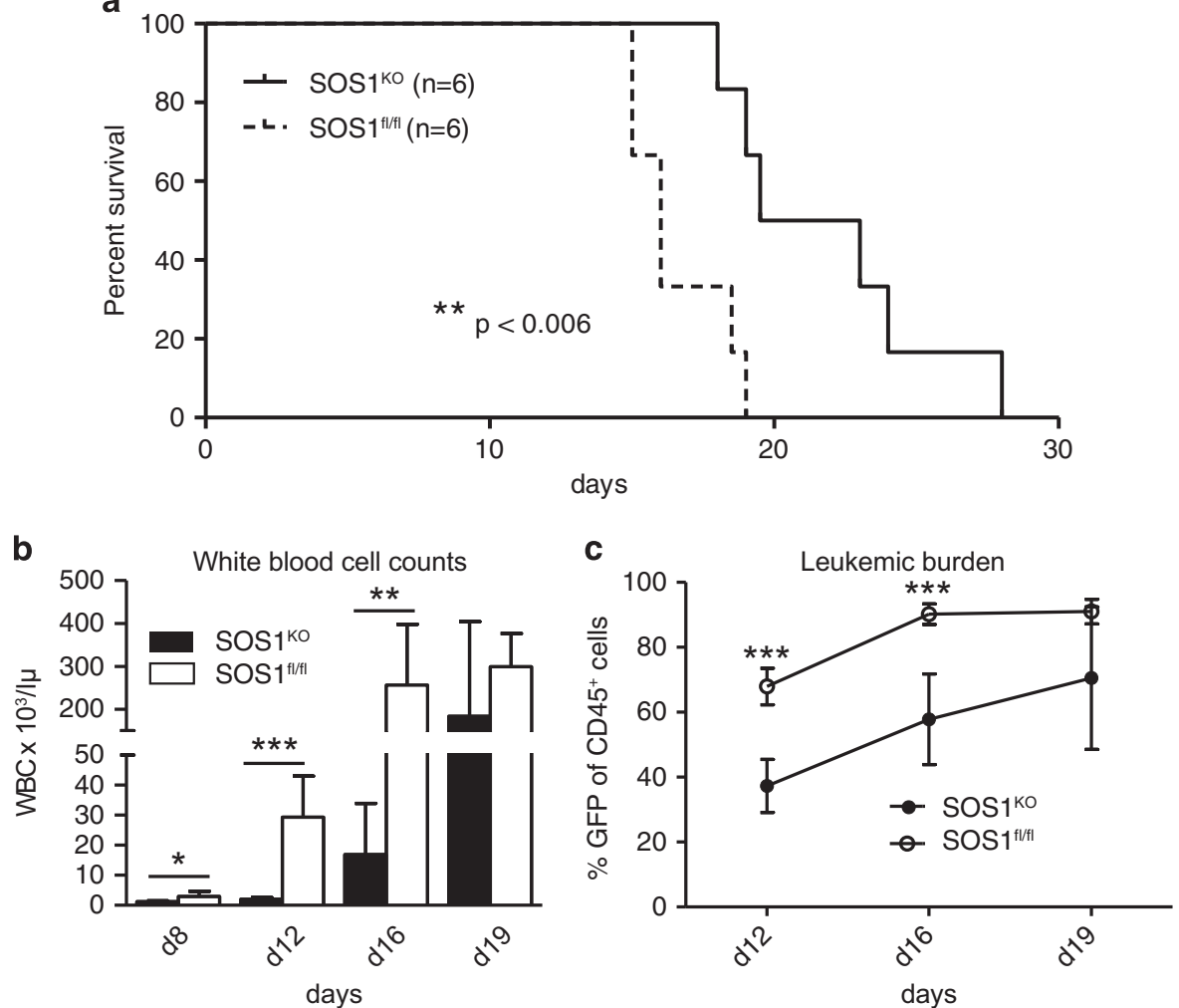

Figure 5. Sos1 deletion abolishes BCR-ABL induced transformation of HSCs in vitro and delays leukemogenesis in vivo. (a) 5-FU enriched BMDCs were retrovirally-infected with pMigBCR-ABL ${ }^{\mathrm{p} 210}$ before being treated with (SOS $1^{\mathrm{KO}}$ ) or without (SOS1 $1^{\mathrm{fl} / \mathrm{fl}}$ ) tamoxifen and transplanted into lethally irradiated recipient mice. Recipient mice were injected i.p. with tamoxifen or solvent for 2 days after TX to induce Sos 1 deletion and monitored for leukemia induction. Tamoxifen had no effect on cell engraftment or leukemic development (not shown). In addition, it did not affect the efficiency of infection, which was about $20 \%$ in the presence or the absence of TX (not shown). Kaplan-Meier plot details the overall survival of transplanted mice $(n=6)$ of 2 independent experiments. P-value was determined by log-rank test. (b and $\mathbf{c}$ ). Peripheral blood measurement of mice that received a Sos1 deleted BMDC transplant showed a significantly reduction in leukemic white blood cells (b) gain, as well as a reduced leukemic burden (c) of Sos1 deleted BMDCs transplanted mice during the course of disease. Results are from a representative transplantation experiment $(n=6)$ out of two independent ones at the indicated time points. Leukemic burden was measured by the percentage of enhanced green fluorescent protein (EGFP)-positive CD45 cells in the PB. $P$-values were determined by Student's $t$-test: ${ }^{*} P<0.05,{ }^{* *} P<0.01$ and ${ }^{* * *} P<0.005$.

recipient mice (Figures 5a-c), without affecting engraftment (Supplementary Figures S9B and C) and stemness potential of the leukemic cells (Supplementary Figures S9E-G).

Collectively, our result supports the notion that SOS1 is phosphorylated by oncogenic BCR-ABL in leukemic cells, is

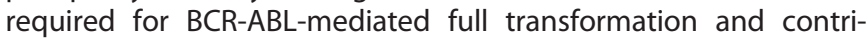
butes to leukemogenesis.

\section{DISCUSSION}

In this work, we provide evidence that SOS1 is phosphorylated by ABL on Y1196. This post-translational modification endows SOS1 with the capacity to activate RAC in response to physiological stimulation of RTKs and in BCR-ABL pathological context. In the former, case an RTK-ABL-pY1196-SOS1 pathway operates to control RAC-mediated actin-based protrusions leading to enhanced directed migration, consistent with a role of $A B L$ and RAC in these processes. ${ }^{42,43}$ A BCR-ABL-pY1196SOS1-RAC axis is instead critical to promote full transforming and leukemogenic ability of the fusion protein.

Our findings are of relevance both for the understanding of the molecular mechanisms through which SOS1 RAC-GEF activity is controlled and further unveil an unexpected role of SOS1-RAC in BCR-ABL transformation. How SOS1 acquires RAC-GEF activity has remained elusive and difficult to reconcile with the finding that the isolated $\mathrm{DH}-\mathrm{PH}$ domain of SOS1 folds into a catalytically inactive conformation. ${ }^{22,37}$ A substantial conformational change of the DH-PH domain must occur for SOS1 to become catalytically proficient. Our findings suggest that phosphorylation of Y1196 may be a way to promote such structural rearrangements. Consistently, we found that the isolated proline-rich region binds directly to the DH domain through residues encompassing Y1196. In addition, phosphorylation of $\mathrm{Y} 1196$ reduces this interaction, induces SOS1 RAC-GEF in vitro and is required to elevate RAC-GTP levels in vivo. Thus, although we cannot exclude that Y1196 might be the binding site of yet-to-be found regulators of SOS1, a plausible possibility is that phosphorylation of this tyrosine is necessary to liberate an inhibitory molecular proline-rich/DH-PH interaction. The latter interaction may not necessarily occur intramolecularly and might drive the formation of inter-molecular interactions. Within this context, ABL-mediated phosphorylation of Y1196 disrupt this linkage, leading to liberation of monomeric SOS1, which may, in turn, become incorporated into large macromolecular complex, necessary for full activation of its RAC1 GEF. Indeed, we have previously shown that one additional way to modulate SOS1 RAC-GEF is via assembly into a macromolecular complex that contains EPS8 and ABI1. ${ }^{12,18,19}$ Intriguingly, the latter protein is an interactor and activator of $\mathrm{ABL}^{44,45}$ suggesting that SOS1 tyrosine phosphorylation may ensue following the assembly of this complex. In keeping with this 
notion, preliminary evidence indicates that $A B L$ coimmunoprecipates with SOS1 and EPS8 in an ABI1-dependent manner. Under this condition, ABL activity is elevated and SOS1 becomes phosphorylated on tyrosine: an event that might be critical for promoting the RAC-GEF activity of the macromolecular complex.

Biochemical and genetic studies in mice models have unequivocally shown that the RAC GTPases are critical downstream signaling targets used by BCR-ABL to promote its full leukemogenic and transforming potential. ${ }^{25,28,29,40,46}$ Mechanistically, the GEFs VAV1, 2 and 3 have been shown to biochemically link BCR$A B L$ to RAC proteins. ${ }^{47}$ Indeed, VAV proteins are tyrosine phosphorylated and activated by ABL. ${ }^{42,47,48}$ Their genetic loss, however, results in complex BCR-ABL-dependent pathological phenotypes and delays, but does not abrogate BCR-ABL leukemogenesis, ${ }^{49}$ suggesting that other GEFs must have important roles. Our findings are consistent with the latter notion by showing that SOS1 is one additional critical exchange factors mediating BCR-ABL-dependent activation of RAC proteins and transforming ability. In none of our assays, we could distinguish whether tyrosine phosphorylated SOS1 displays any preference for the different RAC proteins. However, it would not be unfeasible that different GEFs target specific GTPases (different RACs in this case), in turn required to control a define set of biological outputs. In support of this contention, we noticed, that both RAC2 and VAV3 deficiency reduced cell proliferation and increased apoptosis, possibly interfering with phosphorylation of the pro-apoptotic BAD protein. ${ }^{40,49}$ Loss of Sos 1 instead reduces cell proliferation, but has no effect on apoptosis, while pushing $\mathrm{K} 562$ cells to acquire expression of hemoglobin. K562 cells are bipotent progenitor cells, which are blocked in the differentiation program due to the expression of the BCR-ABL. ${ }^{50}$ Thus, removal of SOS1 may induce these cells to acquire erythrocyte lineage properties, suggesting that SOS1 is required for cell proliferation and possibly for the maintenance of a more undifferentiated state rather than controlling apoptosis.

In summary, our data reveal a novel molecular mechanism through which the poorly studied SOS1 RAC-GEF activity becomes activated by $B C R-A B L$, contributing to its pathogenic, transforming functions. They further suggest that targeting this interaction and signaling axis might be beneficial in combination with $B C R-A B L$ pharmacological treatment to prevent or delay the onset of BCR$A B L$ resistance, at least in part, by reducing the proliferation potential of leukemic progenitors.

\section{CONFLICT OF INTEREST}

The authors declare no conflict of interest.

\section{ACKNOWLEDGEMENTS}

We thank A Bachi (IFOM) for critically reading the manuscript. This work has been supported by the Associazione Italiana per la Ricerca sul Cancro (AIRC 10168 and 18621), the Italian Ministries of Education- University-Research (MIUR-PRIN-2009X23L78), the International Association For Cancer Research (AICR-09-0582 and 14-0335), the CARIPLO Foundation (2010-0737) and the European Research Council (Advanced-ERC268836). ALI was supported by a Grant from der German Jose Carreras Stiftung (DJCLS R14/22) and a Grant from the Government Baden-Württemberg (MWK). CM was supported form a AECC fellowship, Spain.

\section{AUTHOR CONTRIBUTIONS}

SG designed, performed and analyzed data. EF performed and analyzed data. FCB and C Gomez contributed in generating vital new Sos 1 mouse models. AP performed and analyzed data (CRIB assays). SM performed and analyzed mass spectrometry data. JR designed and analyzed mass spectrometry data. FT, C Giuliani and YR performed and analyzed data on K562. GP designed and analyzed ISH data. SK performed and analyzed murine blast data. IP and SM performed and analyzed murine data. MZ and MC knocked down experiments. ES provided key murine models. ALI designed and analyzed primary BMC data. GS designed analyzed data and wrote the manuscript.

\section{REFERENCES}

1 Zhao C, Du G, Skowronek K, Frohman MA, Bar-Sagi D. Phospholipase D2generated phosphatidic acid couples EGFR stimulation to Ras activation by Sos. Nat Cell Biol 2007; 9: 706-712.

2 Gureasko J, Galush WJ, Boykevisch S, Sondermann H, Bar-Sagi D, Groves JT et al. Membrane-dependent signal integration by the Ras activator Son of sevenless. Nat Struct Mol Biol 2008; 15: 452-461.

3 Buday L, Downward J. Epidermal growth factor regulates p21ras through the formation of a complex of receptor, Grb2 adapter protein, and Sos nucleotide exchange factor. Cell 1993; 73: 611-620.

4 Lu TY, Doherty J, Freeman MR. DRK/DOS/SOS converge with Crk/Mbc/dCed-12 to activate Rac1 during glial engulfment of axonal debris. Proc Natl Acad Sci USA 2014; 111: 12544-12549.

5 Nickerson S, Joy ST, Arora PS, Bar-Sagi D. An orthosteric inhibitor of the RAS-SOS interaction. Enzymes 2013; 34(Pt. B): 25-39.

6 Margarit SM, Sondermann H, Hall BE, Nagar B, Hoelz A, Pirruccello M et al. Structural evidence for feedback activation by Ras.GTP of the Ras-specific nucleotide exchange factor SOS. Cell 2003; 112: 685-695.

7 Freedman TS, Sondermann H, Kuchment O, Friedland GD, Kortemme T, Kuriyan J. Differences in flexibility underlie functional differences in the Ras activators son of sevenless and Ras guanine nucleotide releasing factor 1. Structure 2009 14; 17: 41-53.

8 Sondermann H, Soisson SM, Boykevisch S, Yang SS, Bar-Sagi D, Kuriyan J. Structural analysis of autoinhibition in the Ras activator Son of sevenless. Cell 2004; 119: 393-405.

9 Yadav KK, Bar-Sagi D. Allosteric gating of Son of sevenless activity by the histone domain. Proc Natl Acad Sci USA 2010; 107: 3436-3440.

10 Nimnual AS, Yatsula BA, Bar-Sagi D. Coupling of Ras and Rac guanosine triphosphatases through the Ras exchanger Sos. Science 1998; 279: 560-563.

11 Salojin KV, Zhang J, Meagher C, Delovitch TL. ZAP-70 is essential for the T cell antigen receptor-induced plasma membrane targeting of SOS and Vav in T cells. J Biol Chem 2000; 275: 5966-5975.

12 Innocenti M, Tenca P, Frittoli E, Faretta M, Tocchetti A, Di Fiore PP et al. Mechanisms through which Sos-1 coordinates the activation of Ras and Rac. J Cell Biol 2002; 156: 125-136.

13 Yang L, Bashaw GJ. Son of sevenless directly links the Robo receptor to rac activation to control axon repulsion at the midline. Neuron 2006; 52: 595-607.

14 Das B, Shu X, Day GJ, Han J, Krishna UM, Falck JR et al. Control of intramolecular interactions between the pleckstrin homology and Dbl homology domains of Vav and Sos1 regulates Rac binding. J Biol Chem 2000; 275: 15074-15081.

15 Sini P, Cannas A, Koleske AJ, Di Fiore PP, Scita G. Abl-dependent tyrosine phosphorylation of Sos-1 mediates growth-factor-induced Rac activation. Nat Cell Biol 2004; 6: 268-274.

16 Hwang HS, Hwang SG, Cho JH, Chae JS, Yoon KW, Cho SG et al. CIIA functions as a molecular switch for the Rac1-specific GEF activity of SOS1. J Cell Biol 2011; 195: 377-386.

17 Scita G, Nordstrom J, Carbone R, Tenca P, Giardina G, Gutkind S et al. EPS8 and E3B1 transduce signals from Ras to Rac. Nature 1999; 401: 290-293.

18 Scita G, Tenca P, Areces LB, Tocchetti A, Frittoli E, Giardina G et al. An effector region in Eps8 is responsible for the activation of the Rac- specific GEF activity of Sos-1 and for the proper localization of the Rac-based actin-polymerizing machine. J Cell Biol 2001; 154: 1031-1044.

19 Innocenti M, Frittoli E, Ponzanelli I, Falck JR, Brachmann SM, Di Fiore PP et al. Phosphoinositide 3-kinase activates Rac by entering in a complex with Eps8, Abi1, and Sos-1. J Cell Biol 2003; 160: 17-23.

20 Khanday FA, Santhanam L, Kasuno K, Yamamori T, Naqvi A, Dericco J et al. Sosmediated activation of rac1 by p66shc. J Cell Biol 2006; 172: 817-822.

21 Snyder JT, Worthylake DK, Rossman KL, Betts L, Pruitt WM, Siderovski DP et al. Structural basis for the selective activation of Rho GTPases by Dbl exchange factors. Nat Struct Biol 2002; 9: 468-475.

22 Soisson SM, Nimnual AS, Uy M, Bar-Sagi D, Kuriyan J. Crystal structure of the Dbl and pleckstrin homology domains from the human Son of sevenless protein. Cell 1998; 95: 259-268.

23 Daley GQ, McLaughlin J, Witte ON, Baltimore D. The CML-specific P210 bcr/abl protein, unlike v-abl, does not transform NIH/3T3 fibroblasts. Science 1987; 237: 532-535.

24 Kogan SC, Ward JM, Anver MR, Berman JJ, Brayton C, Cardiff RD et al. Bethesda proposals for classification of nonlymphoid hematopoietic neoplasms in mice. Blood 2002; 100: 238-245. 
25 Thomas EK, Cancelas JA, Zheng Y, Williams DA. Rac GTPases as key regulators of p210-BCR-ABL-dependent leukemogenesis. Leukemia 2008; 22: 898-904.

26 Pai SY, Kim C, Williams DA. Rac GTPases in human diseases. Dis Markers 2010; 29: 177-187.

27 Skorski T, Wlodarski P, Daheron L, Salomoni P, Nieborowska-Skorska M, Majewski M et al. BCR/ABL-mediated leukemogenesis requires the activity of the small GTPbinding protein Rac. Proc Natl Acad Sci USA 1998; 95: 11858-11862.

28 Cho YJ, Zhang B, Kaartinen V, Haataja L, de Curtis I, Groffen J et al. Generation of rac3 null mutant mice: role of Rac3 in Bcr/Abl-caused lymphoblastic leukemia. Mol Cell Biol 2005; 25: 5777-5785.

29 Thomas EK, Cancelas JA, Chae HD, Cox AD, Keller PJ, Perrotti D et al. Rac guanosine triphosphatases represent integrating molecular therapeutic targets for BCR-ABL-induced myeloproliferative disease. Cancer Cell 2007; 12: 467-478.

30 Larsen MR, Thingholm TE, Jensen ON, Roepstorff $P$, Jorgensen TJ. Highly selective enrichment of phosphorylated peptides from peptide mixtures using titanium dioxide microcolumns. Mol Cell Proteomics 2005; 4: 873-886.

31 Pinkse MW, Uitto PM, Hilhorst MJ, Ooms B, Heck AJ. Selective isolation at the femtomole level of phosphopeptides from proteolytic digests using 2D-NanoLC-ESI-MS/MS and titanium oxide precolumns. Anal Chem 2004; 76: 3935-3943.

32 Steen H, Kuster B, Mann M. Quadrupole time-of-flight versus triple-quadrupole mass spectrometry for the determination of phosphopeptides by precursor ion scanning. J Mass Spectrom 2001; 36: 782-790.

33 Salek M, Alonso A, Pipkorn R, Lehmann WD. Analysis of protein tyrosine phosphorylation by nanoelectrospray ionization high-resolution tandem mass spectrometry and tyrosine-targeted product ion scanning. Anal Chem 2003; 75: 2724-2729.

34 Pendergast AM, Muller AJ, Havlik MH, Clark R, McCormick F, Witte ON. Evidence for regulation of the human $A B L$ tyrosine kinase by a cellular inhibitor. Proc Nat Acad Sci USA 1991; 88: 5927-5931.

35 Plattner R, Kadlec L, DeMali KA, Kazlauskas A, Pendergast AM. c-Abl is activated by growth factors and Src family kinases and has a role in the cellular response to PDGF. Genes Dev 1999; 13: 2400-2411.

36 Plattner R, Koleske AJ, Kazlauskas A, Pendergast AM. Bidirectional signaling links the Abelson kinases to the platelet-derived growth factor receptor. $\mathrm{Mol} \mathrm{Cell} \mathrm{Biol}$ 2004; 24: 2573-2583.

37 Sondermann H, Soisson SM, Bar-Sagi D, Kuriyan J. Tandem histone folds in the structure of the $\mathrm{N}$-terminal segment of the ras activator Son of Sevenless. Structure 2003; 11: 1583-1593.

38 Hoon JL, Wong WK, Koh CG. Functions and regulation of circular dorsal ruffles. Mol Cell Biol 2012; 32: 4246-4257.

39 Mauro MJ, Druker BJ. STI571: targeting BCR-ABL as therapy for CML. Oncologist 2001; 6: 233-238.
40 Sengupta A, Arnett J, Dunn S, Williams DA, Cancelas JA. Rac2 GTPase deficiency depletes BCR-ABL+ leukemic stem cells and progenitors in vivo. Blood 2010; 116: 81-84.

41 Baltanas FC, Perez-Andres M, Ginel-Picardo A, Diaz D, Jimeno D, Liceras-Boillos P et al. Functional redundancy of Sos1 and Sos2 for lymphopoiesis and organismal homeostasis and survival. Mol Cell Biol 2013; 33: 4562-4578.

42 Daubon T, Chasseriau J, El Ali A, Rivet J, Kitzis A, Constantin B et al. Differential motility of p190bcr-abl- and p210bcr-abl-expressing cells: respective roles of Vav and Bcr-Abl GEFs. Oncogene 2008; 27: 2673-2685.

43 Ridley AJ. Life at the leading edge. Cell 2011; 145: 1012-1022.

44 Ziemnicka-Kotula D, Xu J, Gu H, Potempska A, Kim KS, Jenkins EC et al. Identification of a candidate human spectrin src homology 3 domain- binding protein suggests a general mechanism of association of tyrosine kinases with the spectrin-based membrane skeleton [In Process Citation]. J Biol Chem 1998; 273: 13681-13692.

45 Taki T, Shibuya N, Taniwaki M, Hanada R, Morishita K, Bessho F et al. ABI-1, a human homolog to mouse Abl-interactor 1 , fuses the MLL gene in acute myeloid leukemia with $\mathrm{t}(10 ; 11)(\mathrm{p} 11.2 ; \mathrm{q} 23)$. Blood 1998; 92: 1125-1130.

46 Williams DA, Zheng Y, Cancelas JA. Rho GTPases and regulation of hematopoietic stem cell localization. Methods Enzymol 2008; 439: 365-393.

47 Bustelo XR. Vav family exchange factors: an integrated regulatory and functional view. Small GTPases 2014; 5: 9.

48 Bassermann F, Jahn T, Miething C, Seipel P, Bai RY, Coutinho S et al. Association of $\mathrm{Bcr}-\mathrm{Abl}$ with the proto-oncogene $\mathrm{Vav}$ is implicated in activation of the Rac-1 pathway. J Biol Chem 2002; 277: 12437-12445.

49 Chang KH, Sanchez-Aguilera A, Shen S, Sengupta A, Madhu MN, Ficker AM et al. Vav3 collaborates with p190-BCR-ABL in lymphoid progenitor leukemogenesis, proliferation, and survival. Blood 2012; 120: 800-811.

50 Kang CD, Do IR, Kim KW, Ahn BK, Kim SH, Chung BS et al. Role of Ras/ERKdependent pathway in the erythroid differentiation of K562 cells. Exp Mol Med 1999; 31: 76-82.

c) (i) $\Theta$ This work is licensed under a Creative Commons AttributionNonCommercial-NoDerivs 4.0 International License. The images or other third party material in this article are included in the article's Creative Commons license, unless indicated otherwise in the credit line; if the material is not included under the Creative Commons license, users will need to obtain permission from the license holder to reproduce the material. To view a copy of this license, visit http:// creativecommons.org/licenses/by-nc-nd/4.0/

(c) The Author(s) 2018

Supplementary Information accompanies this paper on the Leukemia website (http://www.nature.com/leu) 